\title{
SEGURANÇA DA TERAPIA MEDICAMENTOSA EM NEONATOLOGIA: OLHAR DA ENFERMAGEM NA PERSPECTIVA DO PENSAMENTO ECOLÓGICO RESTAURATIVO ${ }^{1}$
}

\author{
Gabriela Manito Guzzo², Ana Maria Muller de Magalhães ${ }^{3}$, Gisela Maria Schebella Souto de Moura4 , Wiliam Wegner ${ }^{5}$
}

${ }^{1}$ Artigo extraído da dissertação - Segurança no uso de medicamentos em neonatologia na perspectiva da equipe de enfermagem, apresentada ao Programa de Pós-Graduação em Enfermagem (PPGEnf) da Universidade Federal do Rio Grande do Sul (UFRGS), em 2015.

${ }^{2}$ Mestre em Enfermagem. Enfermeira responsável pelo Gerenciamento de Risco do Hospital Fêmina. Porto Alegre, Rio Grande do Sul, Brasil. E-mail: ggabriela@ghc.com.br

${ }^{3}$ Doutora em Enfermagem. Docente da Escola de Enfermagem da UFRGS. Porto Alegre, Rio Grande do Sul, Brasil. E-mail: amagalhaes@hcpa.edu.br

${ }^{4}$ Doutora em Administração. Docente da Escola de Enfermagem da UFRGS. Porto Alegre, Rio Grande do Sul, Brasil. E-mail: gmoura@hcpa.edu.br

${ }^{5}$ Doutor em Enfermagem. Docente da Escola de Enfermagem da UFRGS. Porto Alegre, Rio Grande do Sul, Brasil. E-mail: wegner@ hcpa.edu.br

\section{RESUMO}

Objetivo: analisar os fatores que interferem na segurança no processo de medicação em uma unidade de terapia intensiva (UTI) neonatal. Método: estudo exploratório com abordagem qualitativa. A coleta ocorreu no período de dezembro de 2014 a março de 2015 , através de grupos focais e caminhada fotográfica, na perspectiva do pensamento ecológico restaurativo, com 12 profissionais de enfermagem da UTI neonatal de um hospital público da Região Sul do Brasil. As informações foram organizadas através do software Nvivo 10 e submetidas à análise de conteúdo temática.

Resultados: a partir da análise, emergiram as seguintes categorias temáticas: Abordagem individualizada e cultura de punição dos erros de medicação; Fatores de (in)segurança relacionados à estrutura física dos medicamentos na UTI neonatal; Fatores de (in)segurança relacionados a rotinas e protocolos; e A enfermagem como barreira para a ocorrência de falhas de prescrição médica.

Conclusão: o estudo demonstra a complexidade do processo de medicação em neonatologia e destaca pontos críticos no mesmo que podem ocasionar falhas e eventos adversos, assim como propostas de melhoria para prevenir os erros. Destaca-se o papel da equipe de enfermagem na detecção de erros da prescrição medicamentosa, funcionando como última barreira para prevenção e redução de erros associados à medicação.

DESCRITORES: Segurança do paciente. Erros de medicação. Neonatologia. Enfermagem neonatal. Unidades de terapia intensiva neonatal.

\section{MEDICATION SAFETY IN NEONATOLOGY: NURSING IN THE PERSPECTIVE OF THE ECOLOGICAL RESTORATIVE APPROACH}

\begin{abstract}
Objective: to analyze the factors that interfere in the medication safety process in a neonatal intensive care unit (NICU).

Method: An exploratory study with a qualitative approach. The collection took place from December 2014 to March 2015, using focus groups and photography walkabouts, in the perspective of the ecological restorative approach, with 12 nursing professionals from the neonatal ICU of a public hospital in the Southern Region of Brazil. The information was organized using the NVivo 10 software and was submitted to thematic content analysis.

Results: the following thematic categories emerged from the analysis : Individualized approach and medication error punishment culture ; Safety factors related to the physical structure of the medications in the neonatal ICU; Safety factors related to routines and protocols; and Nursing as a barrier to the occurrence of medical prescription errors.

Conclusion: the study demonstrates the complexity of the medication process in neonatology and highlights critical points that can cause errors and adverse events, as well as proposals to prevent errors. The role of the nursing team in the detection of medication prescription errors is highlighted, functioning as the last barrier to the prevention and reduction of errors associated with medication.

DESCRIPTORS: Patient safety. Medication errors. Neonatology. Neonatal nursing. Neonatal intensive care units.
\end{abstract}




\title{
SEGURIDAD DE LA TERAPIA MEDICAMENTOSA EN LA NEONATOLOGÍA: UNA MIRADA DE LA ENFERMERÍA EN LA PERSPECTIVA DEL PENSAMIENTO ECOLÓGICO RESTAURATIVO ${ }^{1}$
}

\begin{abstract}
RESUMEN
Objetivo: analizar los factores que interfieren en la seguridad en el proceso de medicación, en una unidad de terapia intensiva (UTI) neonatal. Método: estudio exploratorio y con un abordaje cualitativo. La obtención de datos se dio entre Diciembre del 2014 y Marzo del 2015 a través de grupos de enfoque y caminada fotográfica, en la perspectiva del pensamiento ecológico restaurativo, con 12 profesionales de enfermería de la UTI neonatal de un hospital público de la Región Sur del Brasil. Las informaciones fueron organizadas a través del software Nvivo 10 y sometidas al análisis del contenido temático.

Resultados: a partir del análisis emergieron las siguientes categorías temáticas: Abordaje individualizado y cultura de punición de los errores de medicación, Factores de (in)seguridad relacionados con la estructura física de los medicamentos en la UTI neonatal, Factores de (in)seguridad relacionados con las rutinas y protocolos y La enfermería como barrera para la ocurrencia de fallas de prescripción médica. Conclusión: el estudio demuestra la complejidad del proceso de medicación en la neonatología y destaca puntos críticos, en el mismo, que pueden ocasionar fallas y eventos adversos, así como propuestas de mejoras para prevenir los errores. Se destaca el papel del equipo de enfermería en la detección de errores de la prescripción medica, funcionando como la última barrera para la prevención y reducción de los errores asociados con la medicación.
\end{abstract}

DESCRIPTORES: Seguridad del paciente. Errores de medicación. Neonatología. Enfermería neonatal. Unidades de terapia intensiva neonatal.

\section{INTRODUÇÃO}

A complexidade do sistema de saúde tem aumentado de maneira vertiginosa. O aumento das tecnologias associadas aos tratamentos e cuidados de enfermagem está em constante transformação. Apesar de esses avanços significarem benefícios em diversos aspectos, ainda temos um processo assistencial frágil e com riscos em matéria de segurança, desencadeando efeitos indesejados no cuidado ofertado. ${ }^{1}$

Os efeitos indesejados podem resultar em dano ao paciente, decorrentes de atos intencionais ou não intencionais de profissionais de saúde. ${ }^{2-4}$ Os incidentes que causam danos ao paciente são denominados eventos adversos (EAs), sendo alguns deles consequentes de erros. ${ }^{1-5}$ Os erros são compreendidos como problemas de execução quando não é realizada uma ação planejada, ou problema de planejamento quando um plano errado é aplicado, sendo estes evitáveis. ${ }^{1,5}$

O processo medicamentoso compreende três etapas, são elas: prescrição, dispensação ou distribuição e administração de medicamentos. ${ }^{6}$ Apesar dessa divisão de responsabilidades entre diferentes categorias profissionais, entre elas, médico, farmacêutico e equipe de enfermagem, sabe-se que, na prática, as atividades profissionais acontecem de forma interdependente, e seu sincronismo afeta diretamente a segurança dos pacientes.

Os erros e eventos adversos relacionados à administração de medicamentos estão entre os incidentes mais comuns que podem afetar um paciente nos serviços de saúde, sendo este fato alertado desde o relatório do Instituto de Medicina dos Estados Unidos da América (EUA) publicado em 1999, e permanecendo como uma realidade nos dias atuais. ${ }^{5-7}$

Um em cada sete pacientes adultos hospitalizados experenciam pelo menos um evento adverso relacionado aos cuidados de enfermagem durante sua internação, e os erros de administração de medicamentos estão entre as falhas mais frequentes, ${ }^{8}$ estimando-se que a probabilidade de erros com potencial para causar danos seja três vezes maior em crianças do que em adultos hospitalizados. ${ }^{9}$ Estudo prospectivo de observação sistemática realizado em uma unidade pediátrica no Rio de Janeiro revelou uma taxa de erro de preparo em cerca de $67 \%$ dos medicamentos e $87 \%$ na fase de administração. ${ }^{10}$

Os prematuros com menos de 30 semanas de gestação e menos de $1500 \mathrm{~g}$ são os mais propensos aos eventos adversos, porque estão gravemente enfermos e, por isso, precisam de um número maior de intervenções para sua recuperação, bem como permanecem mais tempo internados. ${ }^{11-14}$ Os erros de medicação, já tão frequentes nos pacientes hospitalizados, têm oito vezes mais chances de ocorrer em UTIs neonatais do que nos demais pacientes internados. ${ }^{13,15}$

Uma recente revisão sistemática objetivou identificar a ocorrência de incidentes com ou sem lesão ocorridos em UTI neonatal descritos na literatura. ${ }^{1} \mathrm{~A}$ pesquisa realizada a partir de 16 artigos, duas dissertações e uma tese, apresentou que os incidentes mais frequentes nessa área estão vinculados a problemas no uso de medicamentos, principalmente relacionados à dosagem incorreta ou inadequada (média de $38 \%$ ), omissão de administração ou falta de prescrição 
de medicamentos necessários, falha de técnica de administração e via de administração errada. ${ }^{1}$

Uma revisão integrativa recente indica a carência de estudos com fortes níveis de evidência no cenário brasileiro em relação a esta temática. ${ }^{16}$ Além disso, observa-se a ausência de pesquisas que permitam melhor compreensão acerca do fenômeno em estudo, indicando a existência de lacunas nessa área do conhecimento.

Frente a esses argumentos e mediante a complexidade do processo de medicação, especialmente nos neonatos, entende-se que a adoção dos pressupostos do pensamento ecológico restaurativo, oferece recursos para explorar de forma abrangente e exaustiva os sistemas sociotécnicos da assistência, com atenção cuidadosa aos fatores locais e sistêmicos desse ambiente de cuidado. ${ }^{17}$ Diante do exposto, o objetivo desta pesquisa constitui em analisar os fatores que interferem na segurança do processo de medicação em uma UTI neonatal.

\section{MÉTODO}

Trata-se de um estudo exploratório, com abordagem qualitativa, realizado na perspectiva do pensamento ecológico restaurativo. O pensamento ecológico restaurativo teve sua gênese no intercâmbio entre as ciências biológicas/naturais e humanas, e foi adaptado para área da saúde e segurança do paciente por pesquisadores canadenses. ${ }^{17-18}$ Essa abordagem proporciona um olhar compreensivo dos processos envolvidos no complexo sistema de saúde atual, por meio do engajamento dos profissionais para explorar, repensar, criticar e propor melhorias tanto nas suas práticas quanto no seu ambiente de trabalho. ${ }^{17-19} \mathrm{O}$ uso de métodos fotográficos, nessa perspectiva, ajuda os profissionais a participar colaborativamente na identificação de riscos e proposição de barreiras para o fortalecimento da segurança do paciente. ${ }^{17-20}$

São considerados os quatro pilares dessa abordagem: (i) a ética do lugar - o trabalho integrado com os membros da comunidade para conhecer sua história, cultura e práticas; (ii) ciência cidadã o emprego de métodos de pesquisa participativos, como observação de campo e métodos fotográficos; (iii) prática engajada - a adoção de práticas restaurativas, automonitoramento e guia de trabalho diário para melhorar as condições do ambiente; e (iv) aprendizagem adaptativa - utilização das descobertas cotidianas para imaginar novamente e recriar melhores maneiras de convívio entre os indivíduos envolvidos e o contexto compartilhado. ${ }^{21}$
O estudo foi realizado na UTI neonatal de um hospital público, especializado na saúde materno-infantil, ao Sul do Brasil. A amostra foi intencional e composta por um enfermeiro e 11 técnicos de enfermagem que estavam trabalhando no período da coleta dos dados, e que, além disso, manifestaram interesse em discutir o tema e participar da pesquisa através da assinatura do Termo de Consentimento Livre e Esclarecido. O critério de inclusão foi ser profissional da equipe de enfermagem na UTI neonatal do local do estudo. Foram considerados critérios de exclusão para a participação da pesquisa: exercício de cargo de chefia do setor na ocasião da coleta de dados, profissionais que atuassem como folguistas (não eram exclusivos da neonatologia), ou que estivessem em contrato temporário, com menos de 6 meses de atuação no setor.

A coleta de dados foi realizada no período de dezembro de 2014 a março de 2015, por meio de dois grupos focais e pelo método fotográfico participativo, técnicas de coleta alinhadas ao pensamento ecológico restaurativo. Participaram da pesquisa o total de 12 profissionais de enfermagem, sendo 11 no primeiro grupo focal, dois deles participaram também da caminhada fotográfica, e quatro deles no segundo grupo focal. No segundo grupo focal houve o ingresso de um novo participante por desistência ou impossibilidade de participação de sete profissionais que estavam no primeiro encontro.

No primeiro grupo focal, participaram 11 profissionais, sendo um enfermeiro e 10 técnicos de enfermagem. Apresentaram-se os objetivos da pesquisa e lançaram-se questões norteadoras para a discussão com base nos dados descritivos dos eventos adversos notificados pelos profissionais da unidade, disponibilizadas pela equipe do gerenciamento de riscos da instituição. As questões norteadoras referiam-se à opinião do grupo sobre o processo de medicação na UTI neonatal, sobre a prática de diluição e administração de drogas injetáveis na unidade, entre outros. Após esse grupo focal, as discussões foram transcritas e analisadas através do programa Nvivo 10, a fim de construir-se um roteiro para a caminhada fotográfica, que visou capturar imagem dos fatores relacionados à segurança no uso de medicação em neonatologia levantados pelos participantes no primeiro grupo focal.

Antes da realização da caminhada fotográfica o roteiro foi validado com três participantes do primeiro grupo focal. A validação deu-se por consenso entre os participantes, sobre a pertinência e relevância dos pontos destacados no roteiro, os quais retratavam as discussões do primeiro grupo 
focal e indicavam pontos críticos do processo de medicação na unidade. Os mesmos foram convidados a participar da caminhada fotográfica, havendo recusa de apenas um dos participantes, por já ter compromisso no dia agendado para a mesma. Os participantes da caminhada fotográfica tiveram a função de narrar as fotos que estavam sendo tiradas, bem como proceder à gravação de todos os diálogos que aconteceram durante o trajeto. Participaram da caminhada fotográfica a pesquisadora principal (orientadora do estudo, guiando a tomada de fotos), a mestranda (tirando as fotos), bolsista de pesquisa (takenoter - realizando as anotações do campo e formulário de fotos digitais), técnico de enfermagem e enfermeira (que conduziam o grupo pela unidade e apontavam os aspectos listados no roteiro), constituindo-se de um grupo de cinco membros.

Após a caminhada fotográfica, as narrativas foram transcritas e analisadas, bem como, as fotografias selecionadas e organizadas pela pesquisadora através do programa Nvivo 10 . Tanto os participantes que tiveram suas falas gravadas, quanto os que foram fotografados, receberam e concordaram com a sua participação através da assinatura de um termo de consentimento livre e esclarecido (TCLE) específico para essa etapa da pesquisa.

Da caminhada fotográfica resultaram 50 fotos, das quais 16 foram selecionadas pela pesquisadora, com base no objetivo do estudo, para analisar os principais fatores que interferiam na segurança do processo medicamentoso no local onde os participantes atuavam, como fonte de discussão do segundo grupo focal, no qual os participantes puderam expressar suas opiniões a respeito das imagens obtidas. Além disso, esse grupo focal objetivou o levantamento das medidas de prevenção aos erros de medicação em neonatologia apontadas pelos participantes desse encontro.

Para a realização do segundo grupo focal foram convidados os mesmos participantes do primeiro. No entanto, sete participantes não puderam se fazer presentes, permanecendo quatro participantes do primeiro encontro, e um membro foi substituído por um suplente da lista de inscritos no segundo grupo focal, totalizando cinco participantes nesse segundo encontro. O número de participantes nos grupos focais seguiu as orientações de estudos que referem de seis a 15 pessoas como recomendável, contudo dimensões menores entre cinco e sete participantes também podem ser utilizadas para aprofundar eterminados aspectos. ${ }^{22-23}$

O material gerado a partir dos grupos focais e caminhada fotográfica foi organizado através do programa NVivo 10 e analisado por meio da análise de conteúdo temática. ${ }^{19}$ Essa metodologia de análise consiste na descoberta dos núcleos de sentido que compõem uma comunicação, cuja presença ou frequência respondam aos objetivos da investigação proposta. ${ }^{24} \mathrm{~A}$ organização dos dados no programa NVivo 10 serviu para ratificar e complementar a categorização proposta pela pesquisadora, dando consistência à análise dos dados.

O estudo foi aprovado pelo Comitê de Ética em Pesquisa sob o número de Protocolo 14.164, CAAE 34641714.7.0000.5530, atendendo às recomendações da Resolução 466/12 do Conselho Nacional de Saúde sobre pesquisa com seres humanos. A pesquisa foi apresentada e autorizada pelos gestores da instituição após a aprovação pelo comitê de ética, e os participantes foram submetidos à um TCLE em cada etapa da pesquisa. Na transcrição das falas foi usada, para fins de identificação dos sujeitos e manutenção da confidencialidade dos mesmos, a letra $\mathrm{P}$ seguida do número referente à sua ordem de participação nos encontros, bem como GF1, GF2 ou $\mathrm{CF}$, distinguindo em qual dos momentos da pesquisa as mesmas aconteceram, grupo focal 1 , grupo focal 2 ou caminhada fotográfica, respectivamente.

\section{RESULTADOS}

As categorias que emergiram da análise de conteúdo temática foram organizadas para melhor compreensão das informações conforme se segue: "Abordagem individualizada e cultura de punição dos erros de medicação", "Fatores de (in)segurança relacionados à estrutura física dos medicamentos na UTI Neonatal", "Fatores de (in)segurança relacionados a rotinas e protocolos" e "A enfermagem como barreira para a ocorrência de falhas de prescrição médica".

\section{Abordagem individualizada e cultura de punição dos erros de medicação}

No primeiro grupo focal, foi discutido como são encaminhadas as medidas punitivas quando ocorrem erros de medicação. Os participantes entendiam que as punições eram feitas de forma diferente dependendo da categoria dos envolvidos, de acordo com a fala que se segue: acho que depende de quem tem que ser punido. Acho que se é um médico as coisas não acontecem. Mas, já, se é um técnico de enfermagem... (P7, GF1).

Ao falar sobre o assunto eles comentaram que, em situações nas quais houve erro do técnico de enfermagem, houve abertura de um processo 
administrativo disciplinar e o profissional foi afastado. No entanto, relataram ocasiões em que médicos haviam cometido falhas, nas quais não havia sido dado nenhum encaminhamento administrativo.

Os erros não eram vistos de maneira sistêmica, e, sim, individualizada, como está descrito nos relatos a seguir sobre um evento adverso sofrido por um bebê em 2013, onde foi instalada uma dieta enteral por via parenteral: isso foi um caso isolado que aconteceu da dieta, da colega acabou de colocar a dieta num acesso venoso (P4, GF2).

Ao reportar essa situação vivenciada, os participantes manifestaram que a profissional envolvida no erro estava voltando de uma licença de saúde prolongada e não tinha condições de assumir integralmente as atividades de enfermagem em uma UTI neonatal. Essa abordagem individualizada do erro foi verbalizada por apenas um participante do segundo grupo focal, mas os demais presentes partilhavam dessa percepção.

\section{Fatores de (in)segurança relacionados à estru- tura física dos medicamentos na UTI Neonatal}

Os participantes discutiram fatores relacionados à estrutura física que tanto contribuem para a segurança do paciente, tal como a segregação dos medicamentos de alta vigilância, quanto predispõem a uma assistência insegura, como, por exemplo, o armazenamento de medicamentos misturados, conforme apresenta a figura 1 a seguir.

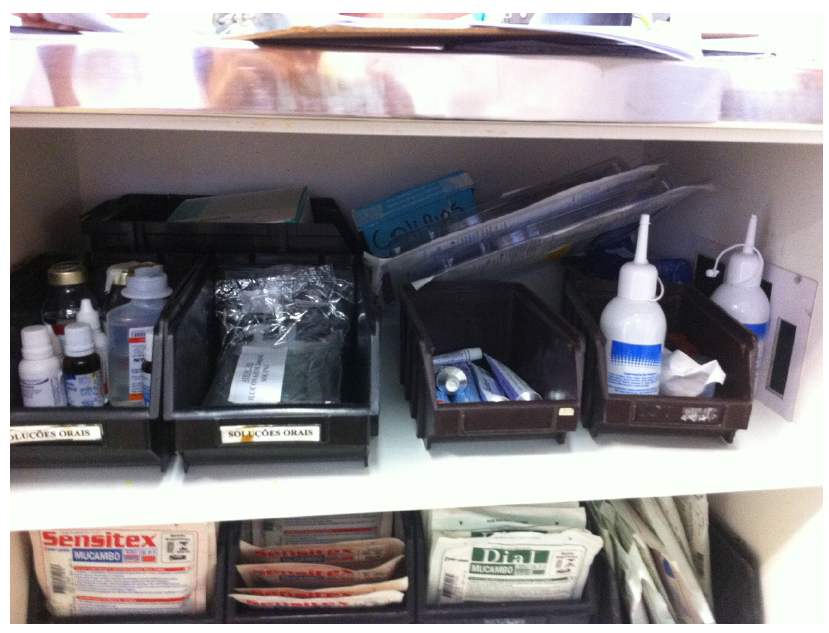

Figura 1 - Foto 35: armário de armazenamento de soluções orais, injetáveis e material médico

Daí tu vai colocar aonde? Aquilo ali é do paciente, a gente não divide com os outros que nem as medicações que ficam no posto. Mas eu vou botar aonde? (P1, GF2).
Na figura 1 identifica-se o acondicionamento de drogas orais e injetáveis junto com materiais médicos e fármacos de uso tópico. Além disso, os medicamentos que não são usados rotineiramente, e não tem em estoque na unidade, mas são prescritos para um paciente, correm o risco de serem perdidos por não haver um lugar específico para o seu armazenamento.

Em contrapartida, os medicamentos controlados, que segundo a rotina da unidade deveriam ficar chaveados, são mantidos abertos, pois junto a eles são armazenadas outras medicações que não pertencem a essa categoria. A figura 2 apresenta a fotografia da gaveta de controlados da unidade estudada.

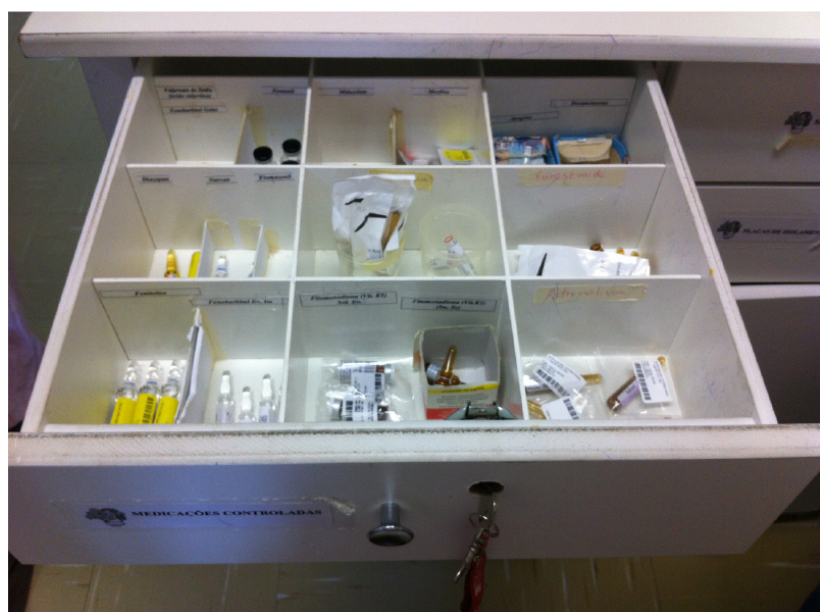

Figura 2 - Foto 4: gaveta de medicamentos controlados

Mas aí tem outras medicações junto que não são controladas (P4, CF).

O risco relacionado aos múltiplos medicamentos armazenados na gaveta de medicações controladas, conforme a Figura 2, foi levantado pelos participantes da caminhada fotográfica e, apesar de não ter sido discutido no primeiro grupo focal, foram realizadas fotografias da mesma. No segundo grupo focal os participantes trouxeram relatos de modificações que tinham ocorrido na gaveta de medicamentos controlados, a partir de sua reorganização pelos próprios profissionais da equipe de enfermagem, no transcorrer da pesquisa. Agora a dos controlados tá só os controlados e tá dividida, cada uma tem um item, e a das medicações agora tá com divisões menores (P2, GF2). 


\section{Fatores de (in)segurança relacionados a rotinas e protocolos}

Os participantes, logo no início do primeiro grupo focal, destacaram a tabela de diluição de medicamentos como sendo uma ferramenta eficaz para a segurança no uso de medicamentos. No entanto, referiram que esta era incompleta para o uso em neonatologia, visto que a diluição para essa população é dependente do peso e outras características do recém-nascido, conforme apresenta a figura 3.

A figura 3 demonstra que a tabela de diluição de medicamentos utilizada no período da pesquisa continha quatro colunas, sendo estas, sequencialmente, o nome da droga, o volume e diluente utilizados para a reconstituição, as condições de armazenamento e o tempo de estabilidade, e alguma observação pertinente ao fármaco. Observa-se que não há uma coluna de diluição ou concentração máxima que oriente o técnico de enfermagem a fazê-la conforme o peso do paciente.

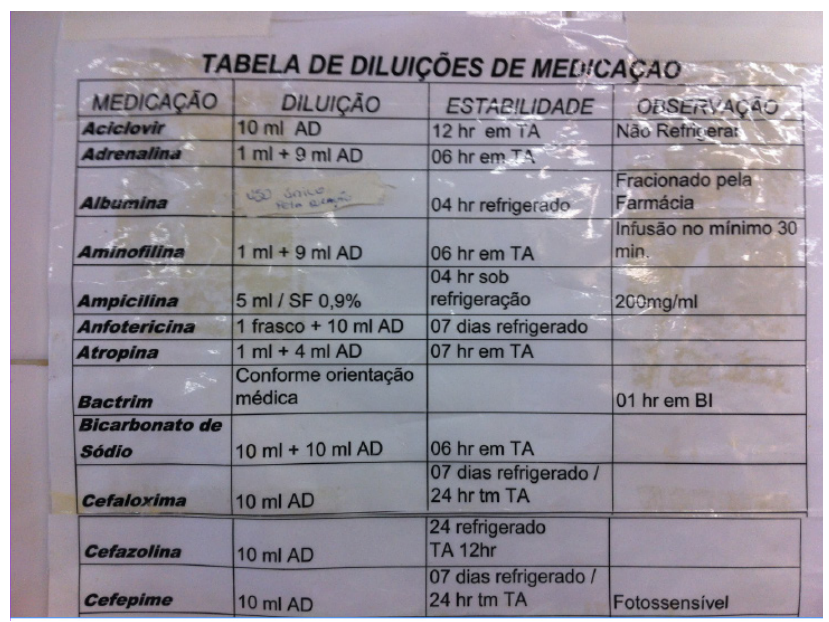

Figura 3 - Foto 46: Tabela de diluição de medicamentos afixada no posto de enfermagem

Para a rediluição a gente não tem uma rotina. [...] E pra fazer num bebê tu vai tirar 0,2 da medicação, tu vai botar mais quanto? O bebê é pequeno, o bebê é grande, tem quantos kg? (P2, GF2). Os medicamentos que possuem estabilidade de algumas horas eram diluídos, identificados e deixados em uma bandeja no posto de enfermagem para uso comum. No entanto, os participantes relataram que essa rotina não era sempre seguida, e que muitas vezes os frascos, ampolas ou flaconetes ficavam abertos nesse local, o que poderia resultar em contaminação do seu conteúdo e consequente dano ao paciente. A figura 4 demonstra o acima exposto.

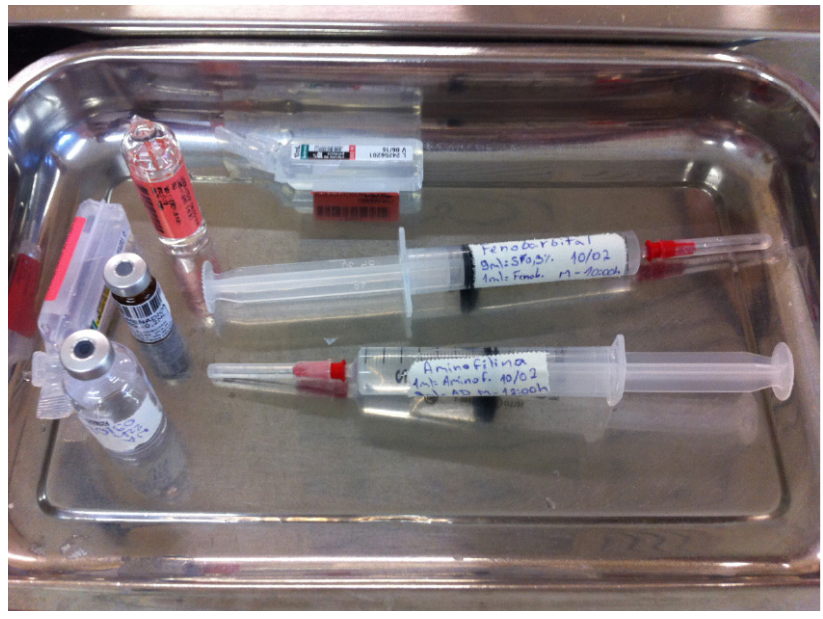

Figura 4 - Foto 2: Bandeja de medicamentos de múltiplas doses no posto de enfermagem

[...] se tu usar um pra cada soro que tu vai fazer lá,é $0,2 \mathrm{ml}$ de $\mathrm{NaCl}$, tu vai pegar e botar outros $20 \mathrm{ml}$ fora. [...] tu acaba deixando ali, porque vai vir daqui a um minuto alguém fazer um soro. Acho que na praticidade tu acaba deixando ali. Só que ao mesmo tempo tu vai deixar ali, tu pode deixar aspirado, né, fica mais seguro (P2, GF2).

Os participantes no primeiro grupo focal falaram bastante sobre o "bandejão", e de como não tinha uma padronização para a disposição desses medicamentos que poderiam ser usados em múltiplos pacientes, como pode ser visualizado na figura 4. Quando a fotografia foi apresentada no segundo grupo focal, foi visível a reprovação dos mesmos ao que estavam vendo. Os participantes relataram que não utilizavam as ampolas ou flaconetes já abertos, nem mesmos as seringas com medicamentos diluídos, conforme a rotina estabelecida no setor, pois pareciam não transmitir segurança.

\section{A enfermagem como barreira para a ocorrência de falhas de prescrição médica}

Neste tópico foram organizadas as falas dos participantes em relação ao papel da equipe de enfermagem como última barreira para que a falha não atinja o paciente, especialmente no que tange à sua atuação como questionadores da prescrição médica. No relato que se segue, os técnicos de enfermagem envolvidos se surpreenderam com as doses prescritas, e questionaram a equipe médica sobre suas prescrições.

Muitas vezes isso acontece. Soros também com quantidades muito elevada de eletrólitos, a gente olha, mas o bebê é muito pequeno, não pode ser. A gente passa pra enfermeira, a enfermeira vai lá no 
médico: 'Não, tá errado, ainda bem que tu viu'. Isso acontece com muita frequência (P6, GF1).

O relato demonstra a preocupação dos técnicos de enfermagem quando doses que não são comuns à sua prática diária aparecem nas prescrições médicas. Ressalta-se que o enfermeiro parece exercer esse papel de ligação, levando as observações dos técnicos de enfermagem e argumentando com a equipe médica.

Em outro caso semelhante relatado, a técnica de enfermagem destacou a importância da autonomia da equipe de enfermagem em fazer uma dupla checagem nos itens que são prescritos, e, especialmente, a liberdade em poder discutir com a equipe médica sobre suas prescrições como forma de prevenir danos desnecessários ao paciente.

[...] eu cheguei na residente e falei com ela: 'Checa, por gentileza, isso aqui, vê se é isso mesmo.' E ela teve a grandeza e a humildade de checar e ver: 'Não, realmente, tá três vezes mais, tá muito alto, eu me enganei' e refez. E eu não apliquei antes, eu fui, não ia aplicar, eu fui tirar a dúvida com ela. Eu acho que isso é importante, a gente ter essa autonomia, essa coisa de poder checar (P9, GF1).

Entretanto, um participante demonstrou preocupação em assumir essa responsabilidade devido ao elevado número de tarefas incluídas no processo de trabalho da enfermagem.

Só que assim, além do nosso trabalho ser carregado por vários fatores, ainda tem que ficar prestando atenção se o médico prescreveu certo. Que tem umas coisas que realmente gritam [...]. Que a gente olha assim: não, isso aqui não é possível, não é isso que é pra fazer. E aí a gente sempre vai e questiona, mas não deveria ser assim, né (P1, GF2).

Os depoimentos dos participantes, conforme as informações descritas e documentadas em fotografias, apontaram diversos riscos potenciais para a segurança do paciente em neonatologia.

\section{DISCUSSÃO}

Os pressupostos da abordagem ecológica restaurativa e dos métodos fotográficos participativos contribuíram para a mobilização dos profissionais envolvidos nos processos de medicação e isso oportunizou um momento rico para que os mesmos pudessem repensar suas práticas e identificar fontes potenciais de riscos para a segurança dos neonatos. Estes aspectos também foram destacados em outros estudos que ressaltam a natureza participativa desta metodologia, a qual, além de modificar os ambientes de prática, contribui para a sustentação das mudanças desejadas, pelo engajamento dos profissionais. ${ }^{17-20}$

Os participantes do estudo relataram a percepção de que as categorias profissionais eram tratadas de forma diferente quando da ocorrência de um evento adverso, e que a enfermagem frequentemente sofria punição, como encaminhamento administrativo de suas falhas. Essa percepção é confirmada em estudo que identificou a persistência da cultura de punição frente aos erros de medicação na equipe de enfermagem. ${ }^{25}$

Nas falas dos participantes podemos perceber a atitude reativa da instituição frente aos eventos adversos ocorridos, e falta de uma política institucional que previna a ocorrência de falhas. $O$ terceiro pilar do pensamento ecológico restaurativo propõe que, através de uma prática engajada, os próprios trabalhadores criem formas de melhorar as condições do meio. ${ }^{21}$ Observa-se um embrião dessa atitude engajada da equipe quando, após a análise de um erro, foram implantadas medidas de barreira para a prevenção das falhas de conexão de vias, com o estabelecimento da rotina de dupla checagem.

A abordagem individualizada do erro, que busca culpabilizar, na maioria das vezes, quem está na assistência direta, não vem dando conta de reduzir as falhas na assistência à saúde. Outras áreas de atividades complexas, tais como a aviação e a indústria, ${ }^{26}$ já utilizam uma abordagem sistêmica para entender o erro, a partir da compreensão de que errar é inerente à atividade humana, visando criar sistemas que antecipem erros e que previnam ou os captem antes que eles causem danos. ${ }^{27}$

A enfermagem atua na última etapa do processo de medicação, através do preparo e administração de drogas. Por isso, tem a oportunidade de desempenhar papel fundamental na detecção de falhas produzidas nas fases de prescrição e dispensação, funcionando como barreira para a consecução de eventos adversos relacionados a fármacos. ${ }^{27-29}$ Isso foi claramente identificado pelos participantes da pesquisa, uma vez que relataram alguns episódios em que tiveram a oportunidade de questionar prescrições médicas inadequadas e evitar que os erros atingissem os pacientes.

Alguns autores, no entanto, identificaram a falta de experiência como um fator de risco para a ocorrência de incidentes relacionados a medicamentos. ${ }^{27,29-30}$ Médicos residentes, enfermeiros e técnicos de enfermagem não familiarizados com prescrições, principalmente em relação a dosagens de medicamentos prescritas para neonatos em terapia intensiva, têm menor probabilidade de reconhecer um erro. 
Além disso, os dados da presente pesquisa demonstraram que o armazenamento em forma de estoque por fármaco na unidade, a perda de medicamentos que não estão entre os de reposição diária, a diluição inadequada e o acondicionamento para uso em mais de um paciente, tanto sob refrigeração quanto na bancada ("bandejão"), tornam o processo inseguro, e predispõem à efetivação do incidente no recém-nascido. Esses achados, que retratam aspectos da ética do lugar, também foram encontrados em revisão sistemática, ${ }^{31}$ visto que outros estudos incluídos na mesma identificaram, principalmente, o estoque de drogas como um fator de risco para troca e perda das mesmas.

A neonatologia, por sua especificidade, exige rotinas direcionadas às necessidades dos recém-nascidos, por isso, protocolos de uso adulto não respondem às demandas assistenciais dessa população. ${ }^{32}$ Os participantes relataram que a tabela de diluição de medicamentos, apesar de ser muito útil para a reconstituição de drogas liofilizadas, não atende à necessidade de padronização da rediluição para a administração de doses muito pequenas, como as usadas pelos pacientes de neonatologia.

As fragilidades e a complexidade dos processos de preparo e administração de medicação estão intrinsicamente relacionadas aos processos de trabalho da equipe de enfermagem. Há necessidade de aprofundamento nessa área do conhecimento, especialmente na área de neonatologia, com vistas a contribuir para a construção da cultura de segurança no cenário da prática. ${ }^{33-34}$

O método de pesquisa ecológico restaurativo prevê que os participantes exerçam uma prática engajada a partir dos resultados que constroem de forma participativa, e uma aprendizagem adaptativa, gerando mudanças em seu meio para aumentar a segurança do paciente. ${ }^{17-19}$ Isso foi percebido ao longo do estudo, pois, ao identificar no primeiro grupo focal e na caminhada fotográfica que os medicamentos controlados estavam armazenados juntamente com outros medicamentos, a própria equipe reorganizou o espaço que comportava essas drogas, e passou a manter essa gaveta fechada. Além disso, a rotina de segregação e controle de medicamentos de alta vigilância foi identificada como ponto de melhoria já implementado para a segurança no uso de medicamentos na unidade, ao que se pode aludir como um processo de educação continuada.

Apesar da relevância dos achados deste estudo, diante da complexidade do tema e do percurso metodológico empregado, há de se considerar as suas limitações. A realização da pesquisa num pe- ríodo limitado de tempo e em uma única instituição restringe a comparação com outras investigações. Além disso, a reduzida participação de enfermeiros e o fato de se ter trabalhado somente com uma categoria profissional circunscrevem os resultados obtidos apenas a uma realidade específica.

A despeito dessas limitações, os resultados apresentam inovações e contribuições para a compreensão do fenômeno, assim como indicativos de ações para a prevenção de riscos para o uso de medicamentos em neonatologia. Somado a isso, vislumbra-se a possibilidade de novas investigações que explorem o assunto em diferentes contextos e entre diferentes categorias profissionais, visto que o processo de medicação inclui também profissionais da área médica e de farmácia. Outro aspecto a ser contemplado, em estudos futuros, é a desafiadora inclusão de usuários (pacientes e familiares), como elementos ativos da segurança no cenário dos serviços de saúde.

\section{CONCLUSÕES}

O estudo da segurança no processo de medicação em neonatologia na perspectiva da equipe de enfermagem, através do pensamento ecológico restaurativo, apresentou-se como uma oportunidade de pensar criticamente sobre este processo, de forma colaborativa, entre pesquisadores e profissionais. Ao perceber a autonomia que a equipe adquiriu para propor e implementar mudanças em sua prática diária de trabalho, foi possível ratificar a importância da investigação voltada para a prática engajada e suas recompensas ao pesquisador em ver sua pesquisa de uma maneira viva.

Os achados apontam a necessidade de revisão das políticas e práticas institucionais no sentido de não centrar a abordagem dos erros na responsabilização individual, sem avaliar as causas sistêmicas que levam ao erro. Além disso, os participantes destacaram o papel da equipe de enfermagem na detecção de erros de prescrição médica, funcionando como última barreira para prevenção de falhas no processo de medicação.

O estudo demonstrou, ainda, que existem inúmeros fatores de risco para incidentes no processo de medicação em neonatologia, tais como a estrutura física relacionada aos fármacos, e a falta ou incompatibilidade de rotinas e protocolos. Entre os fatores relacionados à estrutura física destacam-se as más condições de armazenamento dos fármacos e a guarda de inúmeros medicamentos junto com os controlados da unidade. Já, em relação às rotinas 
e protocolos, os participantes referiram que havia falta de padronização de procedimentos, e que alguns protocolos, tal como a tabela de diluição de medicamentos, eram provenientes do cuidado a adultos, e sua inespecificidade não dá conta das demandas do cuidado a neonatos.

Portanto, o estudo também aponta caminhos para a formação dos profissionais de saúde, enfatizando a segurança no processo medicamentoso como assunto fundamental. Vislumbra-se também que a implementação do pensamento ecológico restaurativo, como uma ferramenta de gestão, pode apoiar os gestores e lideranças dos serviços de saúde na construção de políticas que viabilizem a construção da cultura da segurança do paciente, apoiando os trabalhadores de toda a instituição.

\section{REFERÊNCIAS}

1. Lanzillotti LS, Seta MH, Andrade CLT, Júnior WVM. Eventos adversos e outros incidentes na unidade de terapia intensiva neonatal. Ciênc Saúde Colet [Internet]. 2015 [cited 2016 Mar 10]; 20(3):937-46. Available from: http://www.scielo.br/pdf/csc/ v20n3/pt_1413-8123-csc-20-03-00937.pdf

2. World Health Organization. Conceptual framework for the International Classification for Patient Safety. Genebra: World Health Organization [Internet]; 2009 [cited 2015 Apr 10]. Available from: http://www. who.int/patientsafety/taxonomy/icps_full_report. pdf

3. Ministério da Saúde (BR). Portaria n. 529, de 01 de abril de 2013: institui o Programa Nacional de Segurança do Paciente (PNSP). Brasília (DF): Diário Oficial da União; 2013 [cited 2016 jun 30] Available from: http:/ / www. saude.mt.gov.br/upload/controle-infeccoes/pasta2/ portaria-msgm-n-529-de-01-04-2013.pdf

4. Brasil. Resolução da Diretoria Colegiada n. 36, de 25 de julho de 2013: institui ações de segurança do paciente em serviços de saúde e dá outras providências. Diário Oficial da República Federativa do Brasil [Internet]; 2013 [cited 2016 jun 30]. Available from: http:// www.husm.ufsm.br/janela/legislacoes/segurancapaciente/seguranca-paciente/resolucao-rdc-no-36de-25-de-julho-de-2013.pdf

5. Konh LT, Corrigan JM, Donaldson MS. To err is human: building a safer health care system [Internet]. Washington (DC): Institute of Medicine; 2000 [cited 2015 Oct 20]. Available from: http:/ / www. nap.edu/ openbook.php?record_id=9728\&page $=$ R9

6. Ministério da Saúde (BR). Anexo 3: Protocolo de segurança na prescrição, uso e administração de medicamentos. Brasília (DF): MS, 2013 [cited 2015 jan 15]. Available from: http://www. hospitalsantalucinda.com.br/downloads/prot meficamentos.pdf
7. Duarte SCM, Stipp MAC, Silva MM, Oliveira FT. Eventos adversos e segurança na assistência de enfermagem. Rev Bras Enferm. 2015; 68(1):144-54.

8. D'Amour D, Dubois C, Tchouaket E, Clarke S, Blais $R$. The occurrence of adverse events potentially attributable to nursing care in medical units: cross sectional record review. Inter J Nurs Stud. 2014; 51(6):882-91.

9. Belela ASC, Pedreira MLG, Peterlini MAS. Erros de medicação em pediatria. Rev Bras Enferm. 2011; 64(3):563-9.

10. Baptista SCF, Junior WVM. Análise de erros no processo de preparo e administração de medicamentos em pacientes pediátricos [dissertação]. Rio de Janeiro (RJ): Escola nacional de Saúde Pública Sérgio Arouca, Programa de Pós-graduação em Saúde Pública; 2014.

11. Samra HA, McGrath JM, Rollins W. Patient safety in the NICU: a comprehensive review. J Perinat Neonatal Nurs.2011; 25(2):123-32.

12. Jain $S$, Guglani V. Patient safety in paediatrics and neonatal medication. J Med Research [internet]. 2016 Jan-Feb [cited 2016 Jul 18]; 2(1):16-9. Available from: http://www.medicinearticle.com/JMR_201621_05. pdf

13. Ruiz MTE, Suñol MGM, Miguélez LMR, Ortiz ES, Urroz MI, Camino ML, et al. Los errores de tratamiento en una unidad neonatal, uno de los principales acontecimientos adversos. An Pediatr [internet]. 2016 Apr [cited 2016 Jul 18]; 84(4):211-7. Available from: http://www.sciencedirect.com/ science/article/pii/S2341287916000235

14. Campino A, Arranz C, Unceta M, Sordo B, Pascual P, Lopez-de-Heredia I, et al. Medicine preparation errors in ten Spanish neonatal intensive care units. Eur J Pediatr [internet]. 2016 Feb [cited 2016 Jul 18]; 175:203-10. Available from: http://link.springer.com/ article/10.1007/s00431-015-2615-4

15. Stavroudis TA, Shore AD, Morlock L, Hicks RW, Bundy D, Miller MR. NICU medication errors: identifying a risk profile for medication errors in the neonatal intensive care unit. J Perinatol [Internet].2010 [cited 2015 Mar 12]; 30(7):459-68. Available from: http://www.nature.com/jp/journal/v30/n7/full/ jp2009186a.html

16. Gomes ATL, Assis YMS de, Silva MF, Costa IKF, Feijão AR, Santos VEP. Erros na administração de medicamentos: evidências e implicações na segurança do paciente. Cogitare Enferm. 2016; 21(3):01-11.

17. Marck PB, Kwan JA, Preville B, Raynes M, MorganEckley W, Versluys R. et al. Building safer systems by ecological design: using restoration science to develop a medication safety intervention. Qual Safety Health Care. 2006; 15(2):92-7

18. Gimenes FRE, Marck P, Atila EG, Cassiani SHB. Engaging nurses to strengthen medication safety: fostering and capturing change with restorative photographic research methods. Int J Nurs Pract 
[Internet]. 2014 [cited 2015 Jun 10];13(5):1-8. Available from: http://onlinelibrary.wiley.com/doi/10.1111/ ijn.12304/epdf

19. Marck P, Molzahn A, Berry-Hauf R, Hutchings LG, Hughes S. Exploring safety and quality in a hemodialysis environment with a participatory photographic methods: a restorative approach. Nephrol Nurs J [internet]. 2014 [cited 2015 Jan 14]; 41(1):25-35. Available from: https:// www. healthdesign.org/knowledge-repository/exploringsafety-and-quality-hemodialysis-environmentparticipatory

20. Magalhães AMM, Dall'Agnol CM, Marck PB. Nursing workload and patient safety: a mixed method study with an ecological restorative approach. Rev Latinoam Enferm [Internet]. 2013 [cited 2016 Jan 14]; 21(Spe):146-54. Available from: http://www.scielo. br/pdf/rlae/v21nspe/pt_19.pdf

21. Higgs ES. Nature by design, human agency, natural processes and ecological restoration. Boston: The MIT press; 2003.

22. Ressel LB, Beck CLC, Gualda DMR, Hoffmann RMS, Sehnem GD. O uso do grupo focal em pesquisa qualitativa. Texto Contexto Enferm. 2008;17(4):779-86.

23. Busanello J, Lunardi Filho WD, Kerber NPC, Santos SSC, Lunardi VL, Pohlmann FC. Grupo focal com técnica de coleta de dados. Cogitare Enferm. 2013;18(2):358-64.

24. Minayo MCS, organizador. Pesquisa social: teoria, método e criatividade. 29 ed. Petrópolis (RJ): Vozes; 2010.

25. Dias JD, Mekaro KS, Tibes CMS, Zem-Mascarenhas $\mathrm{SH}$. Compreensão de enfermeiros sobre segurança do paciente e erros de medicação. Rev Min Enferm [Internet]. 2014 [cited 2015 Jul 21]; 18(4):866-73. Available from: http://www.reme.org.br/artigo/ detalhes/969

26. Antonucci R, Porcella A. Preventing medication errors in neonatology: is it a dream? World J Clin Pediatr [Internet]. 2014 [cited 2015 Jan 02]; 3(3):37-44. Available from: https://www.ncbi.nlm.nih.gov/ pmc/articles/PMC4162440

27. Wachter RM. Compreendendo a segurança do paciente. 2 ed. Porto Alegre (RS): AMGH Editora; 2013.
28. Härkänen $M$, Turunen $H$, Saano S, VehviläinenJulkunen K. Detecting medication errors: analysis based on a hospital's incident reports. Int J Nurs Pract [Internet]. 2015 [cited 2016 Jul 04]; 21(2):141-6. Available from: http://onlinelibrary. wiley.com/doi/10.1111/ijn.12227/epdf?r3 referer=wol\&tracking_action=preview_click\&show checkout $=1 \&$ purchase_referrer $=$ onlinelibrary.wiley. com\&purchase_site_license=LICENSE_DENIED

29. Bohomol E. Erros de medicação: estudo descritivo das classes dos medicamentos e medicamentos de alta vigilância. Esc Anna Nery [Internet]. 2014 [cited 2016 Nov 09]; 18(2):311-16. Available from: http:/ / www.scielo.br/scielo.php?script $=$ sci_ arttext\&pid=S1414-81452014000200311

30. Sekar KC. Iatrogenic complications in the neonatal intensive care unit. J Perinatol [Internet]. 2010 [cited 2015 Jun 22]; 30(Spe):S51-6. Available from: http://www.nature.com/jp/journal/v30/n1s/full/ jp2010102a.html

31. Keers RN, Williams SD, Cooke J, Ashcroft DM. Causes of medication administration errors in hospitals: a systematic review of quantitative and qualitative evidence. Drug Saf [Internet]. 2013 [cited 2015 Jan 16];36(11):1045-67. Available from: https://www. ncbi.nlm.nih.gov/pmc/articles/PMC3824584

32. Raju TN, Suresh G, Higgins RD. Patient safety in the context of neonatal intensive care: research and educational opportunities. Pediatr Res [Internet]. 2011 [cited 2015 Feb 11]; 70(1):109-15. Available from: https://www.ncbi.nlm.nih.gov/pmc/articles/ PMC3454497

33. Gonçalves MI, Rocha PK, Anders JC, Kusahara DM, Tomazoni A. Comunicação e segurança do paciente na passagem de plantão em unidades de cuidados intensivos neonatais. Texto Contexto Enferm [Internet]. 2016 [cited 2016 Nov 09]; 25(1):18. Available from: http://www.scielo.br/pdf/tce/ v25n1/0104-0707-tce-25-01-2310014.pdf

34. Silva LD, Camerini FG. Análise da administração de medicamentos intravenosos em hospital da rede sentinela. Texto Contexto Enferm [Internet]. Set. 2012 [cited 2016 Nov 09]; 21(3):633-41. Available from: http://www.scielo.br/scielo.php?script=sci arttext\&pid=S0104-07072012000300019 